\title{
Polarization demultiplexing and optical nonlinearity compensation based on artificial neural networks
}

\author{
Yuichiro Kurokawa, Takeru Kyono, Yuta Fukumoto, \\ Noriki Sumimoto, and Moriya Nakamura ${ }^{\text {a) }}$ \\ School of Science and Technology, Meiji University, \\ 1-1-1 Higashimita, Tama-ku, Kawasaki, Kanagawa 214-8571, Japan \\ a)m_naka@meiji.ac.jp
}

Abstract: We proposed and investigated novel methods for polarization demultiplexing using artificial neural networks (ANNs). It was shown that a three-layer ANN includes butterfly operations and has a polarization-demultiplexing capability. Our proposed cascaded construction of a butterflystructure of FIR filters and an ANN connecting $x$ - and y-polarization components exhibited superior performance in terms of the polarizationdemultiplexing speed. By using the proposed method, optical nonlinearity can also be compensated for at the same time. We examined and compared the performance of the proposed methods by numerical simulations using 10-Gsymbol/s polarization multiplexed 16QAM optical signals.

Keywords: optical communications, optical nonlinearity, polarization multiplexing, digital signal processing, artificial neural network, FIR filter Classification: Fiber-Optic Transmission for Communications

\section{References}

[1] K. Kikuchi, "Digital coherent optical communication systems: Fundamentals and future prospects," IEICE Electron. Express, vol. 8, no. 20, pp. 1642-1662, Oct. 2011. DOI:10.1587/elex.8.1642

[2] K. Kikuchi, "Performance analyses of polarization demultiplexing based on constant-modulus algorithm in digital coherent optical receivers," Opt. Express, vol. 19, no. 10, pp. 9868-9880, May 2011. DOI:10.1364/OE.19.009868

[3] E. Ip and M. Kahn, "Compensation of dispersion and nonlinear impairments using digital backpropagation," J. Lightw. Technol., vol. 26, no. 20, pp. 34163425, Oct. 2008. DOI:10.1109/JLT.2008.927791

[4] Y. Gao, F. Zhang, L. Dou, Z. Chen, and A. Xu, "Intra-channel nonlinearities mitigation in pseudo-linear coherent QPSK transmission systems via nonlinear electrical equalizer," Opt. Commun., vol. 282, no. 12, pp. 2421-2425, 2009. DOI:10.1016/j.optcom.2009.03.002

[5] S. Owaki and M. Nakamura, "Equalization of optical nonlinear waveform distortion using neural-network based digital signal processing," Optoelectronics and Communications Conference (OECC2016), WA2-40, July 2016.

[6] S. Owaki, Y. Fukumoto, T. Sakamoto, N. Yamamoto, and M. Nakamura, "Experimental demonstration of SPM compensation based on digital signal 
processing using a three-layer neural-network for 40-Gbit/s optical 16QAM signal,” IEICE Commun. Express, vol. 7, pp. 13-18, Jan. 2018. DOI:10.1587/ comex.2017XBL0148

[7] J. Estarán, R. Rios-Müller, M. A. Mestre, F. Jorge, H. Mardoyan, A. Konczykowska, J.-Y. Dupuy, and S. Bigo, "Artificial neural networks for linear and non-linear impairment mitigation in high-baudrate IM/DD systems," European Conference on Optical Communication (ECOC2016), M.2.B.2 Sept. 2016.

[8] V. Kamalov, L. Jovanovski, V. Vusirikala, S. Zhang, F. Yaman, K. Nakamura, T. Inoue, E. Mateo, and Y. Inada, "Evolution from 8QAM live traffic to PS 64-QAM with neural-network based nonlinearity compensation on $11000 \mathrm{~km}$ open subsea cable," Optical Fiber Communication Conference (OFC2018), Th4D.5, Mar. 2018.

[9] T. Kyono, Y. Otsuka, Y. Fukumoto, S. Owaki, and M. Nakamura, "Computational-complexity comparison of artificial neural network and Volterra series transfer function for optical nonlinearity compensation with time- and frequency-domain dispersion equalization," European Conference on Optical Communication (ECOC2018), Th2.28, Nov. 2018.

[10] Y. Kurokawa, T. Kyono, Y. Fukumoto, N. Sumimoto, and M. Nakamura, "Polarization demultiplexing and optical nonlinearity compensation based on artificial neural networks and FIR filters," to appear in Optoelectronics and Communications Conference (OECC2019), July 2019.

[11] T. A. Eriksson, H. Bülow, and A. Leven, "Applying neural networks in optical communication systems: Possible pitfalls," IEEE Photon. Technol. Lett., vol. 29, no. 23, pp. 2091-2094, Dec. 2017. DOI:10.1109/LPT.2017.2755663

\section{Introduction}

In digital coherent optical communication systems, digital signal processing (DSP) is playing an important role in realizing not only carrier-phase estimation but also polarization demultiplexing and linear compensation of waveform distortion caused by, e.g., chromatic dispersion and polarization-mode dispersion (PMD) [1, 2]. Optical nonlinearity compensation in the digital domain is regarded as the next significant step in compensating for nonlinear effects such as self-phase modulation (SPM) and cross-phase modulation (XPM). Some algorithms have been investigated to realize a digital nonlinear equalizer, including digital back propagation (DBP) and the Volterra series transfer function (VSTF) [3, 4]. However, one drawback is the enormous amount of calculations required, which causes time delays and increases the power consumption at the receiver. Artificial neural networks (ANNs) have been investigated as another candidate which can realize digital nonlinear equalizers to compensate for optical nonlinear effects $[5,6,7,8]$. One important merit of using an ANN is its ability to decrease the computational complexity of the nonlinear equalizer [9]. In those studies, ANN-based nonlinear equalization has been performed on single-polarization signals or signals after a polarization demultiplexing process. In our study, we clarified that polarization demultiplexing can be performed using an ANN because an ANN includes butterfly operations [10]. We investigated novel methods using ANNs to perform polarization demultiplexing and nonlinear compensation at the same time. In this paper, 
we compared the performance of these methods under various conditions of input optical power to the optical fiber. The investigation revealed that our proposed cascaded construction of a butterfly-structure of FIR filters and an ANN connecting $\mathrm{x}$ - and $\mathrm{y}$-polarization components had superior performance in terms of the polarization-demultiplexing speed.

\section{Polarization demultiplexing and nonlinear compensation}

In a digital coherent receiver, polarization demultiplexing is performed by using butterfly-structured FIR filters [1, 2]. However, it can be considered that polarization demultiplexing can also be performed using an ANN because an ANN includes butterfly structures. Here, we compare an FIR filter and a two-layer ANN. Fig. 1(a) shows an FIR filter. Delayed signals are multiplied by filter coefficients and summed. The same operation is performed in a two-layer ANN with a tapped delay line, as shown in Fig. 1(b). The unit in the output layer performs a weighted sum of the signals from the input-layer units. Figs. 1(c) and (d) show butterflystructured FIR filters and a two-layer ANN with two-channel tapped delay lines, respectively. In Fig. 1(c), $h_{x x}, h_{x y}, h_{y x}$, and $h_{y y}$ show FIR filters in the butterfly structure. Blue lines and red lines denote the connections from the x-port and y-port, respectively. The same operation is performed in the two-layer ANN, as shown in Fig. 1(d). The two units in the output layer of the ANN output demultiplexed $\mathrm{x}$ - and y-polarization components of the signal.

Figs. 2(a) to (d) show four methods. We compared the polarization demultiplexing and optical nonlinearity compensation performance of these methods. Fig. 2(a) shows a simple butterfly structure of FIR filters, which is generally used for polarization demultiplexing (method 1). Fig. 2(b) shows a conventional method (method 2), in which an independent ANN compensates for the optical nonlinearity of each polarization component after polarization demultiplexing. However, one drawback of this method is that the independent ANNs cannot compensate for XPM between the two polarization components because of the lack of information interchange between the demultiplexed polarization components. Fig. 2(c) shows

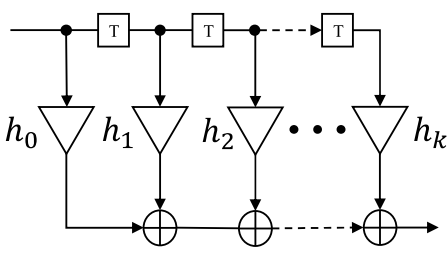

(a) FIR filter.

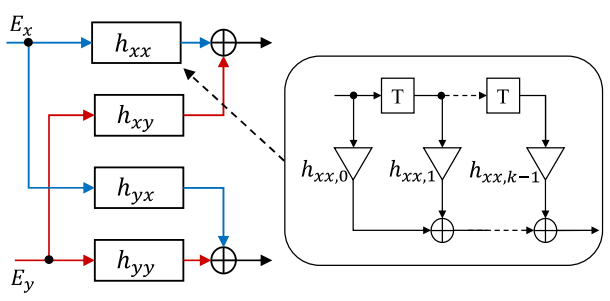

(c) Butterfly-structured FIR filter.

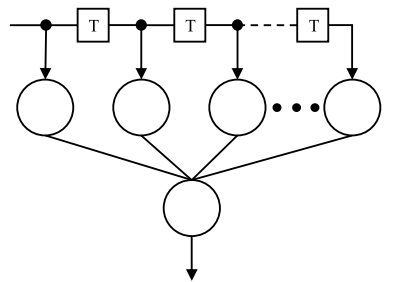

(b) Two-layer ANN.

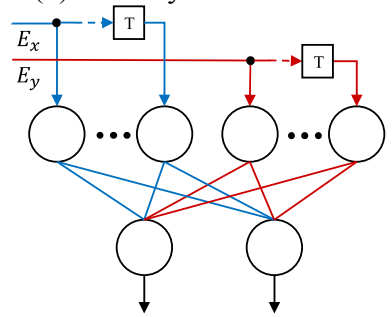

(d) Two-layer ANN with 2-input channels.

Fig. 1. FIR filters and ANNs. 
our proposed novel method (method 3), in which one ANN compensates for the nonlinear waveform distortion of the two polarization components after the polarization demultiplexing. Fig. 2(d) shows another proposed novel method (method 4), in which an ANN works for polarization demultipexing and optical nonlinearity compensation at the same time.

\section{System setup}

Fig. 2(e) shows an optical-fiber transmission system used in our simulations. A 10-Gsymbol/s polarization multiplexed 16QAM optical signal was modulated by PRBS $2^{11}-1$ data and transmitted by a $20-\mathrm{km}$ standard single mode fiber (SSMF). The wavelength of the laser diode (LD) was $1552.5 \mathrm{~nm}$. The noise figure of the Er-doped fiber amplifier (EDFA) was $3 \mathrm{~dB}$. A polarization controller (PC) was used to adjust the state of polarization (SOP) of the transmitted optical signal. The optical signal was received by optical homodyne detection using an optical $90^{\circ}$ hybrid and balanced photodetectors (BPDs). The power of the local oscillator was $3 \mathrm{dBm}$. Here, we assumed that the LO was ideally synchronized to the optical signal. For polarization demultiplexing and nonlinear compensation, DSP based on the four methods in Fig. 2(a) to (d) was performed. The number of hidden layer of each ANN in the methods was set to 15 . The number of taps was set to 11 . The

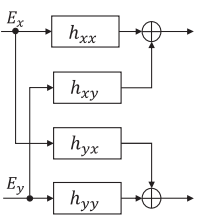

(a) Method 1 .

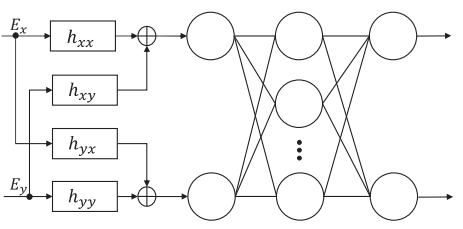

(c) Method 3 .

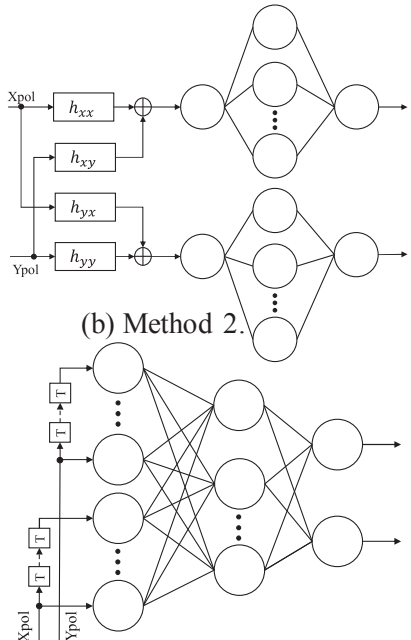

(d) Method 4.

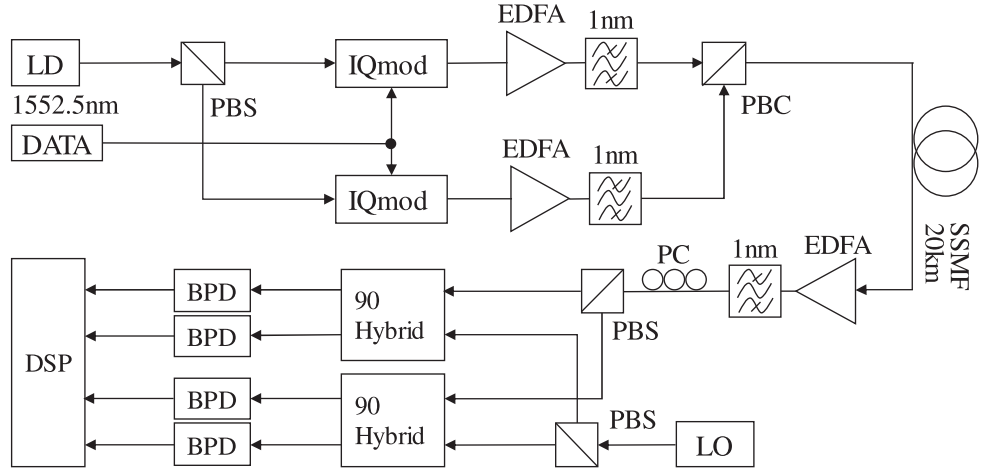

(e) System setup of polarization multiplexing 16QAM transmission. 
ANNs and the FIR filters were trained by a least mean squares (LMS) algorithm using PRBS $2^{11}-1$ data. We confirmed that over-training did not occur, by testing the results using some other PRBS data such as $2^{9}-1$ and $2^{15}-1$, which is needed to eliminate overestimation [11].

\section{Results and discussion}

First, we adjusted the input power to the SSMF to $0 \mathrm{dBm}$ for the x-polarization and $12 \mathrm{dBm}$ for the y-polarization. In this case, SPM is observed only on the ypolarization component. On the other hand, we can observe XPM only on the x-polarization. Fig. 3(a) shows the constellations of the $x$ - and y-polarizations of the received signal without any DSP. The waveform was completely distorted by polarization crosstalk, optical nonlinearity and dispersion. Fig. 3(b) shows the constellations of the $\mathrm{x}$ - and $\mathrm{y}$-polarization components after the processing using method 1. In this case, polarization demultiplexing and dispersion compensation were performed by the FIR filters. However, XPM on the x-polarization and SPM on the y-polarization could not be compensated for. Fig. 3(c) shows the constellations of the $\mathrm{x}$ - and y-polarizations after the processing using method 2. In this case, SPM on the y-polarization component was compensated for by the ANN for the y-polarization. However, XPM on the x-polarization could not be compensated for because the ANN for the x-polarization did not have any information about the y-polarization signal. Fig. 3(d) shows the constellations of the $\mathrm{x}$ - and y-polarizations after the processing using method 3. In this case, both XPM on the x-polarization and SPM on the y-polarization could be compensated for. Fig. 3(e) shows the constellations of the $\mathrm{x}$ - and $\mathrm{y}$-polarizations after the processing using method 4. In this case, the polarization demultiplexing and the compensation of XPM and SPM were successfully performed as well. There was no significant difference between the compensation performance of method 3 and that of method 4. Figs. 3(f) and (g) show the mean-square errors of the $\mathrm{x}$ - and $\mathrm{y}$-polarization signals versus the number of iterations of the learning steps, respectively. We performed the learning processes 10 times for each method, while changing the SOP of the incoming signals and the random initial values of the parameters of the ANNs and the FIR filters. By employing method 3 or 4 , the error was successfully minimized on both the $\mathrm{x}$ - and y-polarizations. However, it should be noted that the convergence speed of the learning of method 3 was faster than that of method 4. Next, we adjusted the input power to the SSMF to $12 \mathrm{dBm}$ for both the $\mathrm{x}$ - and $\mathrm{y}$-polarizations. In this case, the optical signals on both the $\mathrm{x}$ - and $\mathrm{y}$ polarizations are distorted by SPM and XPM. Fig. 3(h) shows the constellations before DSP. Fig. 3(i) shows the constellations after the processing of method 1. The nonlinear distortions caused by SPM and XPM were not compensated for. Fig. 3(j) shows the constellations after the processing of method 2. In this case, only the effect of SPM was compensated for by the ANNs. However, XPM was not compensated for. Figs. 3(k) and (1) show the constellations after the processing of methods 3 and 4, respectively. In these cases, the polarization demultiplexing and the nonlinear compensation of SPM and XPM were successfully performed. Fig. 3(m) shows the mean-square errors of the signals on the x-polarization versus 


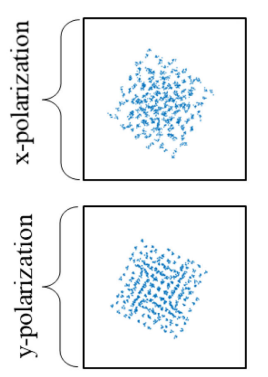

(a) Before compensation.

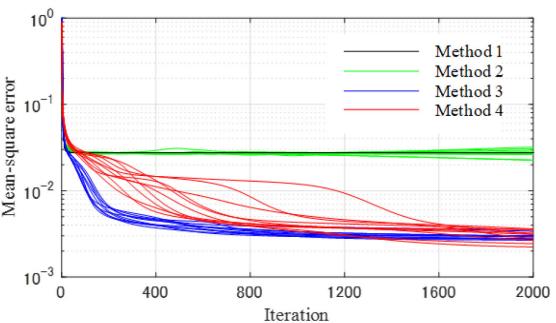

(f) Mean-square error versus learning process iterations (x-polarization)
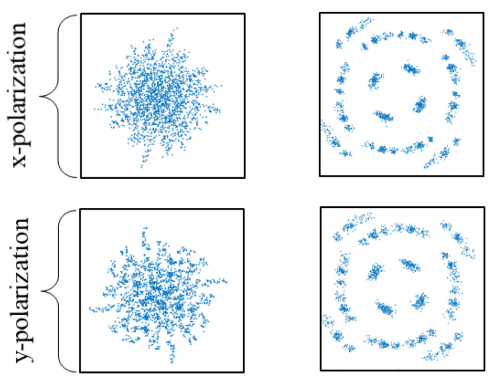

(h) Before compensation.

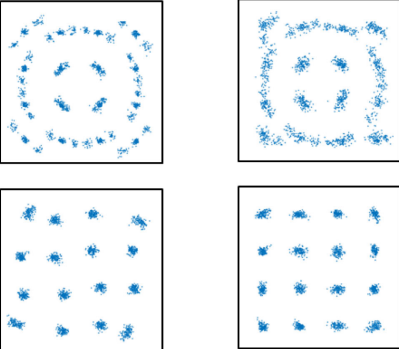

(c) Method 2.
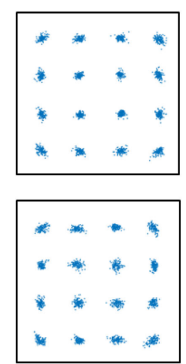

(d) Method 3 .

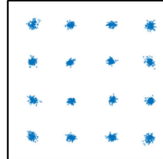

$*$ * *

- *

$+*$

Method 4

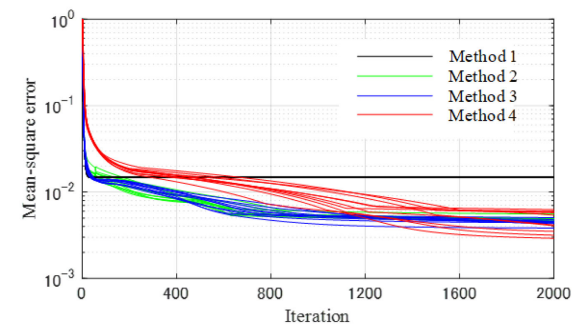

(g) Mean-square error versus learning process iterations (y-polarization).
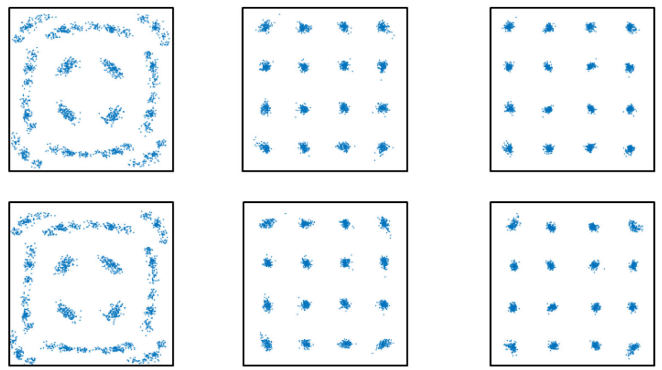

(j) Method 2

(k) Method 3

(1) Method 4.

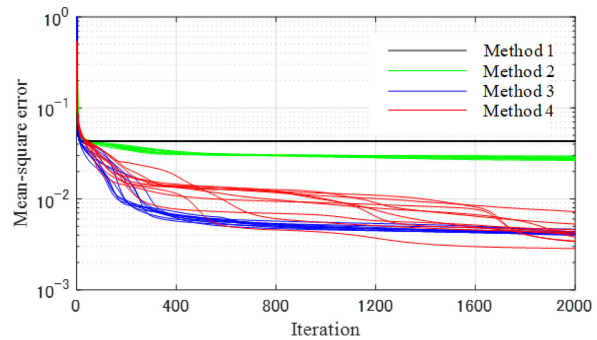

(m) Mean-square error versus learning process iterations (x-polarization).

Fig. 3. Constellations and learning speed.

the number of iterations of the learning steps. Also in this case, the convergence speed of the learning of method 3 was faster than that of method 4. From the results above, we conclude that the performance of method 3 is superior to method 4 from the viewpoint of polarization demultiplexing speed.

\section{Conclusion}

We investigated methods for polarization demultiplexing and nonlinear compensation using ANNs. We showed that an ANN could perform polarization demultiplexing and nonlinear compensation at the same time. Our proposed cascaded construction of a butterfly-structure of FIR filters and an ANN connecting $\mathrm{x}$ - and y-polarizations showed superior performance in terms of polarization demultiplexing speed and optical nonlinearity compensation. 